\title{
Clara Cell Protein and Surfactant Protein D Plasma Levels are Associated with Clinical Outcomes of Mechanically Ventilated Patients
}

\author{
Determann RM ${ }^{1,2,3 *}$, Royakkers AANM4, Lutter $\mathbf{R}^{5,6}$, Korevaar JC ${ }^{7}$ and Schultz MJ1,3 \\ ${ }^{1}$ Department of Intensive Care Medicine, Academic Medical Center, Amsterdam, The Netherlands \\ ${ }^{2}$ Department of Internal Medicine, Academic Medical Center, Amsterdam, The Netherlands \\ ${ }^{3}$ Laboratory of Experimental Intensive Care Medicine and Anesthesiology (L.E.I.C.A.), The Netherlands \\ ${ }^{4}$ Department of Anesthesiology, Tergooi Ziekenhuizen, Location Blaricum, Blaricum, The Netherlands \\ ${ }^{5}$ Department of Experimental Immunology, Academic Medical Center, Amsterdam, The Netherlands \\ ${ }^{6}$ Department of Pulmonology, Academic Medical Center, Amsterdam, The Netherlands \\ ${ }^{7}$ Department of Biostatistics and Epidemiology, Academic Medical Center, Amsterdam, The Netherlands
}

\section{Abstract}

Background: Plasma levels of Clara cell protein (CC16) and Surfactant Protein D (SP-D) are elevated in patients with Acute Lung Injury or Acute Respiratory Distress Syndrome (ALI/ARDS). We investigated the relation between these biomarkers and clinical outcome scores from mechanically ventilated patients at risk for lung injury.

Methods: Data from all 150 patients enrolled in a previously reported preventive randomized controlled trial, comparing a $10 \mathrm{ml} / \mathrm{kg}$ with a $6 \mathrm{ml} / \mathrm{kg}$ tidal volume strategy in patients without ALI/ARDS at the onset of mechanical ventilation, were used. CC16 and SP-D levels were measured in plasma samples at baseline and on day 2 and 4 after initiation of the mechanical ventilation protocol. The relation between CC16 and SP-D levels and development of ALI/ARDS (North American European Consensus Conference (NAECC) criteria), and of the following clinical scores: lung injury score, Sequential Organ Failure Assessment (SOFA) score, and oxygenation index, was investigated using multivariate regression analysis.

Results: Plasma CC16 and SP-D levels increased after 4 days in patients who developed acute lung injury (NAECC criteria). At all time points the plasma CC16 level was significantly correlated with the lung injury score, SOFA score and oxygenation index. The highest correlations were observed on day 2 (standardized coefficient, $\beta=0.38$; $\beta=0.54$; and $\beta=0.40 ; P<0.001$ for all, respectively). The systemic SP-D level was correlated with these scores only on day 4 ( $\beta=0.29 ; \beta=0.26$; and $\beta=0.33 ; P<0.05$ for all, respectively).

Conclusion: Plasma CC16 and SP-D levels may be used to monitor the extent of lung injury in mechanically ventilated intensive care unit patients.

Keywords: Acute respiratory distress syndrome; Tidal volume; Biomarker, Clara cell protein; Surfactant protein D

Abbreviations: CC16: Clara Cell Protein; SP-D: Surfactant Protein D; ALI/ARDS: Acute Lung Injury/Acute Respiratory Distress Syndrome; SOFA: Sequential Organ Failure Assessment; PEEP: Positive End-Expiratory Pressure

\section{Introduction}

Acute Lung Injury (ALI) and its more severe form Acute Respiratory Distress Syndrome (ARDS) are major causes of morbidity and mortality in Intensive Care Unit (ICU)-patients [1]. A correct and well-timed diagnosis of ALI/ARDS allows the clinician to apply specific measures such as reduction of tidal volume size and restrictive fluid management $[2,3]$. Both the lung injury score [4] and the North-American European Consensus Conference criteria [5] are commonly employed to define patients with ALI/ARDS. More recently, an alternative definition, i.e. the Delphi definition, has been proposed [6]. The applied criteria are, however, susceptible to personal interpretation, potentially leading to gross under-recognition [7]. In addition, the $\mathrm{PaO}_{2} / \mathrm{F}_{\mathrm{i}} \mathrm{O}_{2}$-ratio and chest radiograph abnormalities can be influenced by use of higher levels of Positive End-Expiratory Pressure (PEEP) so that patients may no longer fulfill the ALI/ARDS criteria [8].

Adding objective measures such as biological markers could improve recognition of patients with ALI/ARDS. In a recent study we showed that plasma levels of Clara cell protein (CC16) and surfactant protein D (SP-D) are diagnostic of ALI/ARDS in patients with ventilator-associated pneumonia [9]. Thus, biomarkers as CC16 and SP-D may aid in the (early) recognition of ALI/ARDS.
In the present study, we aimed to extend these findings by investigating the relationship between plasma levels of Clara cell protein (CC16), surfactant protein D (SP-D) and clinical scores relevant to ALI/ARDS patients. In a recently reported study in patients without ALI/ARDS at the onset of mechanical ventilation we showed that reduction of tidal volume size may prevent ALI/ARDS during the course of mechanical ventilation [10]. Moreover, with higher tidal volumes (10 $\mathrm{ml} / \mathrm{kg}$ predicted body weight) an increased lung injury score was observed after 4 days, indicative of the presence of lung injury. In the present study we determined plasma CC16 and SP-D levels and related these findings to lower and conventional tidal volumes and development of acute lung injury (NAECC criteria) during the course of mechanical ventilation. Furthermore, we studied whether levels of these biomarkers were independently associated with the lung injury score, sequential organ failure score, and oxygenation index and development lung injury during the course of mechanical ventilation.

*Corresponding author: Determann RM, Department of Intensive Care Medicine Academic Medical Center Meibergdreef, 91105 AZ, The Netherlands, Tel: 00-3120-5669111; Fax: 00-31-20-5669568; E-mail: r.m.determann@amc.uva.nl

Received December 28, 2012; Accepted February 21, 2013; Published February 25, 2013

Citation: Determann RM, Royakkers AANM, Lutter R, Korevaar JC, Schultz M (2013) Clara Cell Protein and Surfactant Protein D Plasma Levels are Associated with Clinical Outcomes of Mechanically Ventilated Patients. J Pulmon Resp Med 3 : 138. doi:10.4172/2161-105X.1000138

Copyright: ( 2013 Determann RM, et al. This is an open-access article distributed under the terms of the Creative Commons Attribution License, which permits unrestricted use, distribution, and reproduction in any medium, provided the original author and source are credited. 
Citation: Determann RM, Royakkers AANM, Lutter R, Korevaar JC, Schultz MJ (2013) Clara Cell Protein and Surfactant Protein D Plasma Levels are Associated with Clinical Outcomes of Mechanically Ventilated Patients. J Pulmon Resp Med 3: 138. doi:10.4172/2161-105X.1000138

Page 2 of 4

Finally we explored the diagnostic accuracy of these markers for the diagnosis of lung injury.

\section{Methods}

\section{Study design/patient selection}

Data from 150 patients participating in a previously reported randomized controlled trial of tidal volume of $10 \mathrm{ml} / \mathrm{kg}$ versus $6 \mathrm{ml} /$ $\mathrm{kg}$ predicted body weigh in patients without ALI/ARDS at the onset of mechanical ventilation were used [10]. The protocol was approved by the institutional review board of both hospitals where the trial was performed and written informed consent was obtained as previously described. For the present study, plasma samples from all patients enrolled were available for analysis of CC16 and SP-D levels.

\section{Trial-related procedures}

All study methods have been previously reported in detail, including inclusion and exclusion criteria and mechanical ventilation procedures. In short, patients were randomly assigned to mechanical ventilation with tidal volumes of 10 or $6 \mathrm{ml} / \mathrm{kg}$ predicted body weight. The volume-controlled mode was used according to the study protocol until the patient could be weaned from the ventilator. Development of lung injury was scored by two independent intensivists who had access to all clinical data except ventilator settings. The lung injury score was calculated including the static lung compliance. The Sequential Organ Failure Assessment (SOFA) score was calculated as described previously [11]. The oxygenation index was calculated using the following formula: mean airway pressure $\times \mathrm{FiO}_{2} \times 100 \% / \mathrm{PaO}_{2}[12]$.

\section{Measurement of biomarkers}

Levels of CC16 and SP-D were measured in plasma samples which were collected at baseline (day 0), on day 2 and day 4. CC16 and SP-D levels were measured with an enzyme-linked immunosorbent assay as described previously [9] but the exception that we used a different capture antibody for the SP-D assay (clone HM3022, HyCult, Uden, The Netherlands).

\section{Statistics}

Data are presented as medians $\left(25^{\text {th }}\right.$ percentile $-75^{\text {th }}$ percentile interquartile ranges). Because the CC16 and SP-D levels were not normally distributed the bivariate analyses were performed with the Mann Whitney U test. The relations between CC16 and SP-D levels with the lung injury score, SOFA score and oxygenation index were studied with the Spearman's correlation coefficient for each time point. In case age was significantly correlated with these variables in univariate analysis, a linear regression model was constructed to study the relation while controlling for age.

To study whether plasma CC16 and SP-D levels change over time in patients ventilated with lower or conventional tidal volumes a linear mixed model was constructed by adding time and randomization group as factors in the model. In this model, the interaction between time and randomization was used to assess any effect of lower tidal volumes on CC16 and SP-D levels. To study changes over time in relation to development of lung injury we constructed the same model but now with time and development of lung injury as factors in the model. To study the relation between baseline CC16 and SP-D levels and development of lung injury, a logistic regression model was constructed with randomization group and biomarker levels as independent variables. Receiver operating characteristic curves were constructed for CC16 and SP-D levels on day 2 and 4 to study the diagnostic accuracy to distinguish patients who did and did not develop lung injury.
All analyses were performed with SPSS version 15.0 (Chicago, Illinois, USA). $P$ value $<0.05$ was considered as statistically significant.

\section{Results}

The patient population was as previously described [10]. In short, 74 patients were randomized to mechanical ventilation with conventional tidal volumes and 76 patients to lower tidal volumes. Twelve patients developed ALI/ARDS. Ten of these patients were randomized to conventional tidal volumes. There were no significant differences between both randomization groups with regard to baseline characteristics and presence of risk factors for ALI/ARDS (Table 1). The median $\left(25^{\text {th }}\right.$ percentile- $75^{\text {th }}$ percentile IQR) CC16 plasma levels in patients ventilated with conventional and lower tidal volumes at baseline were $10.4(5.2-21.6) \mathrm{ng} / \mathrm{ml} v$ s. $8.8(5.6-18.9) \mathrm{ng} / \mathrm{ml}$, respectively $(\mathrm{P}=0.68)$ and SP-D plasma levels were $286(159-518) \mathrm{ng} / \mathrm{ml}$ vs. 396 $(236-528) \mathrm{ng} / \mathrm{ml}$, respectively $(\mathrm{P}=0.10)$. There was evidence for a lower $\mathrm{CC} 16$ level in smokers but statistical significance was not reached. The CC16 level in non-smokers was $12.0(5.9-31.0)$ and in smokers $(8.6$ (4.6-17.7), $\mathrm{P}=0.09$. In both randomization groups the CC16 levels did

\begin{tabular}{|l|c|c|c|}
\hline & $\begin{array}{c}\mathbf{V}_{\mathbf{T}} \text { of } \mathbf{1 0} \mathbf{~ m l} / \mathbf{k g} \\
(\mathbf{n = 7 4 )}\end{array}$ & $\begin{array}{c}\mathbf{V}_{\mathbf{T}} \text { of } \mathbf{6 ~ m l} / \mathbf{k g} \\
(\mathbf{n = 7 6 )}\end{array}$ & $\boldsymbol{P}$-value \\
\hline Age (years) & $58( \pm 17)$ & $63( \pm 15)$ & 0.06 \\
\hline Male sex & $50(68 \%)$ & $49(64 \%)$ & 0.69 \\
\hline APACHE-II score & $20( \pm 8)$ & $21( \pm 7)$ & 0.93 \\
\hline SOFA & $8( \pm 4)$ & $7( \pm 3)$ & 0.19 \\
\hline LIS & $1.2( \pm 0.6)$ & $1.3( \pm 0.6)$ & 0.08 \\
\hline Underlying ALI risk factors: & & & 0.60 \\
\hline -Sepsis & 7 & 4 & \\
\hline -Shock & 6 & 9 & \\
\hline -Pneumonia & 1 & 3 & \\
\hline -Aspiration & & 1 & \\
\hline -Pancreatitis & 12 & 10 & 0.47 \\
\hline -Trauma & 14 & 11 & 0.72 \\
\hline Blood transfusion & 6 & 5 & 0.04 \\
\hline Chronic alcohol abuse & $45(61 \%)$ & $58(76 \%)$ & 0.68 \\
\hline Smoking history & $10.4(5.2-21.6)$ & $8.8(5.6-18.9)$ & 0.10 \\
\hline Baseline CC16 (ng/ml) & $286(159-518)$ & $396(236-528)$ & \\
\hline Baseline SP-D (ng/ml) & & & \\
\hline & & & \\
\hline
\end{tabular}

$V_{T}$ tidal volume; APACHE-II acute physiology and chronic health evaluation-I score; SOFA sepsis organ failure assessment score; LIS lung injury score; CC16 Clara cell protein; SP-D surfactant protein D

Table 1: Baseline characteristics.
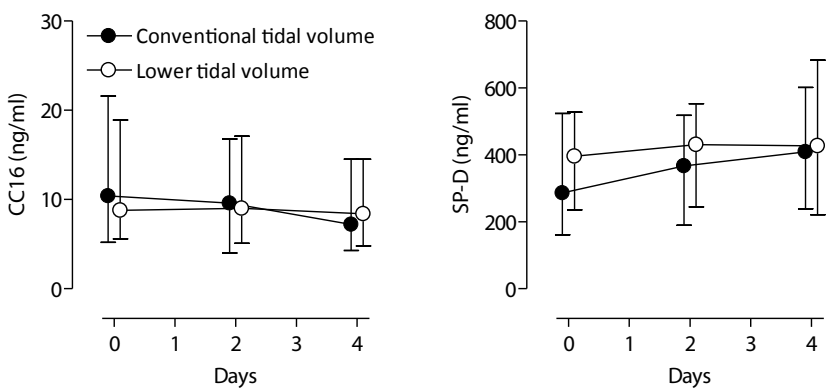

Figure 1: Serial data on plasma levels of Clara cell protein (CC16) and surfactant protein $D(S P-D)$ in patients ventilated with conventional tidal volumes of $10 \mathrm{ml}$ per kilogram of predicted body weight (closed circles) and patients ventilated with lower tidal volumes of $6 \mathrm{ml}$ per kilogram of predicted body weight (open circles). 
not change over time but the SP-D levels increased slightly after 4 days in the conventional tidal volume group $(\mathrm{P}=0.02$; Figure 1$)$.

With univariate analysis, the plasma CC16 levels were significantly correlated with the oxygenation index, the lung injury score and the SOFA score at all three time points. The strongest correlations were observed on day 2: oxygenation index, $\rho=0.36$, lung injury score, $\rho=0.34$ and SOFA score, $\rho=0.51(\mathrm{P}<0.001$ for all). On day 2 and 4 the SP-D levels were significantly correlated with the SOFA score, but only on day 4 a significant correlation with the lung injury score and oxygenation index was observed. With multivariate analysis controlling for age, the correlations (standardized beta) mostly remained significant (Table 2).

The baseline plasma CC16 and SP-D levels were not significantly different between patients who developed lung injury and those who did not. At study entry CC16 levels were 12.0 (8.3-26.6) ng/ml vs. 9.6 (5.3-20.0) ng/ml $(\mathrm{P}=0.35)$ and SP-D levels were $278(190-590) \mathrm{ng} /$ $\mathrm{ml}$ vs. $330(178-514) \mathrm{ng} / \mathrm{ml}(\mathrm{P}=0.98)$ in patients who did and did not develop lung injury respectively. CC16 levels decreased significantly after 4 days in patients who did not develop lung injury $(\mathrm{P}<0.001)$ while levels increased slightly in lung injury patients (Figure 2). This difference was significantly different between groups (linear mixed model, interaction $\mathrm{P}=0.006$; Figure 2). By day 4 the $\mathrm{SP}-\mathrm{D}$ levels were also significantly increased in patients with lung injury and this increase was also significantly different from patients who did not develop lung injury $(\mathrm{P}<0.001$; Figure 2).

In logistic regression analysis, both the baseline CC16 and SP-D level were not predictive of development of lung injury. At day 2 however, both CC16 and SP-D levels were significantly higher in lung injury patients. The diagnostic properties of both biomarkers were moderate as shown by the area under the curve of the receiver operating curves (CC16: $\mathrm{AUC}=0.68$ [95\% CI 0.54-0.82], $\mathrm{P}=0.04$;

\begin{tabular}{|l|c|c|c|}
\hline & Day 0 & Day 2 & Day 4 \\
\hline CC16 & & & \\
\hline Oxygenation index & 0.12 & $0.41^{* * *}$ & $0.24^{*}$ \\
\hline Lung injury score & $0.22^{*}$ & $0.39^{* * *}$ & $0.29^{*}$ \\
\hline SOFA score & $0.37^{* * *}$ & $0.53^{* * *}$ & $0.50^{\star * *}$ \\
\hline SP-D & & & \\
\hline Oxygenation index & 0.02 & 0.06 & $0.33^{*}$ \\
\hline Lung injury score & 0.07 & 0.06 & $0.29^{*}$ \\
\hline SOFA score & 0.02 & $0.22^{*}$ & $0.26^{*}$ \\
\hline
\end{tabular}

CC16 Clara cell protein; SP-D surfactant protein D; SOFA sequential organ failure assessment; ${ }^{*} P<0.05,{ }^{* *} P<0.01,{ }^{* * *} P<0.001$.

Table 2: Standardized coefficients (beta) of multivariate linear regression analysis.
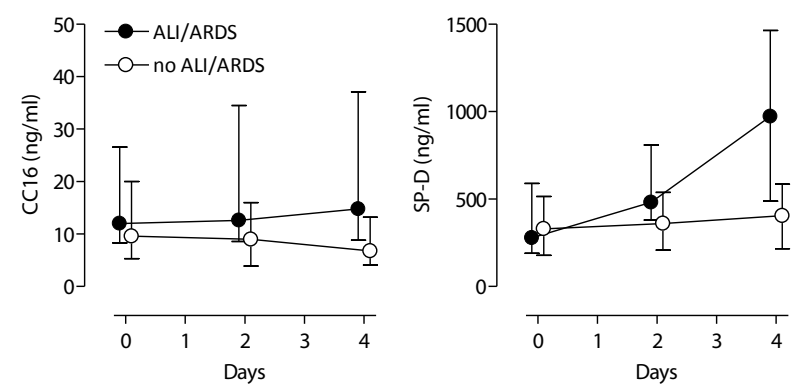

Figure 2: Serial data on plasma levels of Clara cell protein (CC16) and surfactant protein $D(S P-D)$ in patients who developed acute lung injury according to the North American European Consensus Conference criteria (closed circles) and in patients who did not develop acute lung injury (open circles).
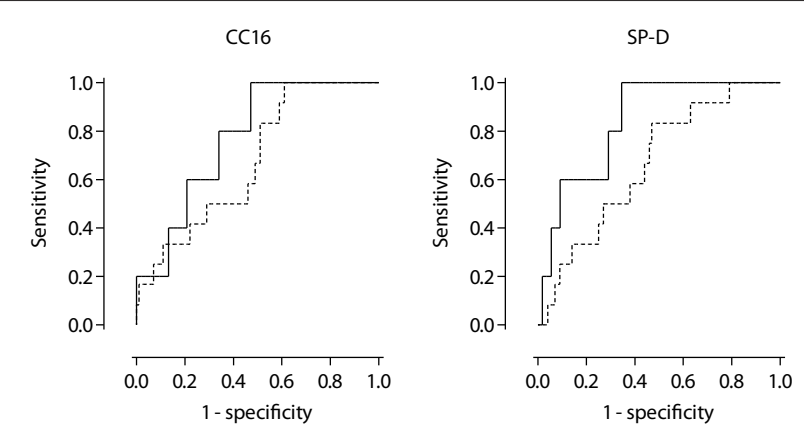

Figure 3: Receiver operating characteristic curves of plasma levels of Clara cell protein (CC16) and surfactant protein D (SP-D) for the differentiation between patients with and without acute lung injury on day 2 (dashed line) and day 4 (straight line).

SP-D: AUC=0.66 [95\% CI 0.52-0.81], P=0.06, Figure 3). By day 4, the diagnostic characteristics had improved markedly. The area under the curve for CC16 was 0.77 (95\% CI 0.60-0.94; P=0.04) and for SP-D 0.84 (95\% CI $0.70-0.98 ; \mathrm{P}=0.01$ ). A cut-off value of $8 \mathrm{ng} / \mathrm{ml}$ of CC16 had a sensitivity of $100 \%$ (95\% CI $82-100$ ) but specificity was low with $54 \%$ (95\% CI 47-61). A cut-off value of $450 \mathrm{ng} / \mathrm{ml} \mathrm{SP-D} \mathrm{yielded} \mathrm{a} \mathrm{sensitivity}$ of $100 \%$ (95\% CI $82-100)$ and a specificity of $65 \%$ (95\% CI $65-58)$.

\section{Discussion}

Several conclusions can be drawn from the present study. First, we observed that plasma levels of CC16 correlate well with the lung injury score, oxygenation index and SOFA score at days 0,2 and 4 . Second, we also observed a correlation between plasma SP-D levels and these scores, but only after 4 days of mechanical ventilation. Third, development of lung injury in this cohort of patients was accompanied by a 3-fold increase in plasma SP-D levels while CC16 levels were only moderately increased in patients with lung injury. And finally, although both CC16 and SP-D levels increased after lung injury had been diagnosed, the diagnostic accuracy of these biomarkers was only moderate.

CC16 is a low-molecular weight protein $(16 \mathrm{kDa})$ that is produced by Clara cells in the small airways [13]. SP-D is a large (589 $\mathrm{kDa})$, asymmetric protein [14] produced by alveolar type II cells and Clara cells [13]. Accumulation of these lung proteins into blood is determined by the leakiness of the respiratory membrane as well as by the gradient for each protein across the respiratory membrane. For a large protein like SP-D, passage of the respiratory membrane is limited and thus a steeper gradient will affect its passage across the respiratory membrane less than that of a small protein like CC16. Thus enhanced plasma levels of SP-D provide in particular an indication of damage to the respiratory membrane. In contrast, elevated plasma levels of CC16 may be caused by both an enhanced local production as well as increased leakiness across the respiratory membrane. In the present study we observed an increase in CC16 levels but not as high as we expected based on our observations in patients with ventilator-associated pneumonia [9] and by others [15-18]. Based on CC16 plasma levels one cannot distinguish between an enhanced leakiness and enhanced local production of CC16. Interestingly, the increase in plasma SP-D levels was much more profound than the increase in CC16 levels. As leakage of SP-D across the respiratory membrane is more restricted than that of $\mathrm{CC} 16$, the increased accumulation of SP-D in the blood is highly suggestive of more damage of the respiratory membrane in patients with lung injury, although this does not preclude an altered SP-D production. In rats challenged with intratracheal lipopolysaccharide, bronchoalveoalar 
SP-D levels were increased after 72 hours [19]. In contrast, in patients with ALI/ARDS, bronchoalveolar SP-D levels remained comparable to control levels [20]. Whether an enhanced SP-D production is manifest in our study group remains to be determined.

We observed a strong correlation between the SOFA score and CC16 and a moderate correlation between the lung injury score and CC16 on all 4 days but a correlation with SP-D only on the fourth day. The delay in correlation is probably caused by the same reasons mentioned above. CC16 may enter the bloodstream more readily than SP-D. Systemic SP-D levels probably do not increase before the permeability has increased significantly. Mechanical ventilation may induce lung injury at a rate that manifests in increased SP-D levels only after a couple of days. Surprisingly the correlation of CC16 with the SOFA score was much stronger than with the lung injury score. The SOFA score is calculated as a score based on objective biomarkers of organ dysfunction [11]. The lung injury score is partly dependent on the interpretation of chest radiographs, which can be subjective. This may explain the weaker correlation. Multiple organ failure is often accompanied by respiratory failure. The strong relation of plasma CC16 levels with the SOFA score may be an indication that CC16 in plasma is a good candidate biomarker to monitor injury to the lungs, even in the absence of all the criteria for ALI/ARDS.

We have demonstrated before that CC16 is an excellent biomarker for ALI/ARDS in patients with ventilator-associated pneumonia [9]. In contrast, the diagnostic accuracy of CC16 plasma levels was only moderate in the present study while the diagnostic accuracy of SP-D was comparable to what we previously observed in patients with ventilator-associated pneumonia [9]. This may be due to a high number of patients with congestive heart failure which is recently shown to be associated with high CC16 levels [21]. The rate of SP-D production may be comparable between patients with pneumonia and other causes of ALI/ARDS while pneumonia may have a different impact on CC16 release compared to sepsis or trauma. The absolute levels of SP-D we observed in the present study were comparable to the levels in patients with ventilator-associated pneumonia and ALI/ARDS of the previous study. This may indeed explain the same level of diagnostic accuracy of SP-D in both our studies.

In the present study we observed that plasma levels of CC16 and SP-D increase in patients with lung injury. We observed that plasma CC16 levels correlate strongly with the SOFA score and moderately with the lung injury score and oxygenation index in mechanically ventilated patients. The diagnostic accuracy of these biomarkers was however moderate. This may be related to different underlying risk factors for ALI/ARDS. Whether differences in CC16 production or transfer to the bloodstream exist between patients with direct or indirect causes of ALI/ARDS remains to be investigated in future studies.

\section{Acknowledgement}

The study was designed by RMD, RL and MJS. Acquisition of the data was performed by RMD, AANMR and RL. Analysis and interpretation of data was done by RMD, JK, and MJS. The manuscript was drafted by RMD, RL, and MJS. Critical revision of the manuscript for important intellectual content was done by $R M D$, AANMR, RL, JK and MJS. The statistical analysis was done by RMD, JK, and MJS. Final approval of the manuscript was done by RMD, AANMR, RL, JK and MJS RMD had full access to all of the data in the study and he takes full responsibility for the integrity of all of the data and the accuracy of the data analysis, including and especially any adverse effects.

\section{References}

1. Rubenfeld GD, Herridge MS (2007) Epidemiology and outcomes of acute lung injury. Chest 131: 554-562.
2. Girard TD, Bernard GR (2007) Mechanical ventilation in ARDS: a state-of-theart review. Chest 131: 921-929.

3. Wheeler AP, Bernard GR (2007) Acute lung injury and the acute respiratory distress syndrome: a clinical review. Lancet 369: 1553-1564.

4. Murray JF, Matthay MA, Luce JM, Flick MR (1988) An expanded definition of the adult respiratory distress syndrome. Am Rev Respir Dis 138: 720-723.

5. Bernard GR, Artigas A, Brigham KL, Carlet J, Falke K, et al. (1994) The American-European Consensus Conference on ARDS. Definitions, mechanisms, relevant outcomes, and clinical trial coordination. Am J Respir Crit Care Med 149: 818-824.

6. Ferguson ND, Davis AM, Slutsky AS, Stewart TE (2005) Development of a clinical definition for acute respiratory distress syndrome using the Delphi technique. J Crit Care 20: 147-154.

7. Ferguson ND, Frutos-Vivar $F$, Esteban $A$, Fernández-Segoviano $P$, Aramburu JA, et al. (2005) Acute respiratory distress syndrome: underrecognition by clinicians and diagnostic accuracy of three clinical definitions. Crit Care Med 33: 2228-2234

8. Estenssoro E, Dubin A, Laffaire E, Canales HS, Sáenz G, et al. (2003) Impact of positive end-expiratory pressure on the definition of acute respiratory distress syndrome. Intensive Care Med 29: 1936-1942.

9. Determann RM, Millo JL, Waddy S, Lutter R, Garrard CS, et al. (2009) Plasma CC16 levels are associated with development of ALI/ARDS in patients with ventilator-associated pneumonia: a retrospective observational study. BMC Pulm Med 9: 49

10. Determann RM, Royakkers A, Wolthuis EK, Vlaar AP, Choi G, et al. (2010) Ventilation with Lower Tidal Volumes as Compared with Conventional Tidal Volumes for Patients without Acute Lung Injury: A Preventive Randomized Controlled Trial. Crit Care 14: R1

11. Arts DG, de Keizer NF, Vroom MB, de Jonge E (2005) Reliability and accuracy of Sequential Organ Failure Assessment (SOFA) scoring. Crit Care Med 33 1988-1993.

12. Trachsel D, McCrindle BW, Nakagawa S, Bohn D (2005) Oxygenation index predicts outcome in children with acute hypoxemic respiratory failure. Am J Respir Crit Care Med 172: 206-211.

13. Hermans C, Bernard A (1999) Lung epithelium-specific proteins: characteristics and potential applications as markers. Am J Respir Crit Care Med 159: 646678

14. Crouch E, Persson A, Chang D, Heuser J (1994) Molecular structure of pulmonary surfactant protein D (SP-D). J Biol Chem 269: 17311-17319.

15. Bernard A, Hermans C, Van Houte G (1997) Transient increase of serum Clara cell protein (CC16) after exposure to smoke. Occup Environ Med 54: 63-65.

16. Broeckaert F, Arsalane K, Hermans C, Bergamaschi E, Brustolin A, et al. (2000) Serum clara cell protein: a sensitive biomarker of increased lung epithelium permeability caused by ambient ozone. Environ Health Perspect 108: 533-537.

17. Michel O, Murdoch R, Bernard A (2005) Inhaled LPS induces blood release of Clara cell specific protein (CC16) in human beings. J Allergy Clin Immunol 115: 1143-1147.

18. Lesur O, Langevin S, Berthiaume Y, Légaré M, Skrobik Y, et al. (2006) Outcome value of Clara cell protein in serum of patients with acute respiratory distress syndrome. Intensive Care Med 32: 1167-1174.

19. Mclntosh JC, Swyers AH, Fisher JH, Wright JR (1996) Surfactant proteins A and $\mathrm{D}$ increase in response to intratracheal lipopolysaccharide. Am J Respir Cell Mol Biol 15: 509-519.

20. Greene KE, Wright JR, Steinberg KP, Ruzinski JT, Caldwell E, et al. (1999) Serial changes in surfactant-associated proteins in lung and serum before and after onset of ARDS. Am J Respir Crit Care Med 160: 1843-1850.

21. Kropski JA, Fremont RD, Calfee CS, Ware LB (2009) Clara cell protein (CC16), a marker of lung epithelial injury, is decreased in plasma and pulmonary edema fluid from patients with acute lung injury. Chest 135: 1440-1447. 